\title{
EchoGéo
}

$58 \mid 2021$

Varia

\section{Voyage au cœur du monde du béton en Afrique}

Entretien avec Armelle Choplin réalisé par Serge Weber en

visioconférence le 6 décembre 2021.

Armelle Choplin et Serge Weber

\section{OpenEdition}

Journals

Édition électronique

URL : https://journals.openedition.org/echogeo/22582

DOI : $10.4000 /$ echogeo.22582

ISSN : 1963-1197

Éditeur

Pôle de recherche pour l'organisation et la diffusion de l'information géographique (CNRS UMR 8586)

Référence électronique

Armelle Choplin et Serge Weber, « Voyage au cœur du monde du béton en Afrique », EchoGéo [En ligne], 58 | 2021, mis en ligne le 31 décembre 2021, consulté le 23 février 2022. URL : http:// journals.openedition.org/echogeo/22582 ; DOI : https://doi.org/10.4000/echogeo.22582

Ce document a été généré automatiquement le 23 février 2022.

EchoGéo est mis à disposition selon les termes de la licence Creative Commons Attribution - Pas d'Utilisation Commerciale - Pas de Modification 4.0 International (CC BY-NC-ND) 


\title{
Voyage au cœur du monde du béton en Afrique
}

\author{
Entretien avec Armelle Choplin réalisé par Serge Weber en
} visioconférence le 6 décembre 2021.

\section{Armelle Choplin et Serge Weber}

\section{RÉFÉRENCE}

\section{Armelle Choplin}

Matière grise de l'urbain. La vie du ciment en Afrique.

2020. Genève, MétisPresses, $252 \mathrm{p}$.

Lien vers la version numérique en PDF : https://archive-ouverte.unige.ch/unige:145150

Serge Weber (SW). Quelle a été la genèse de ce livre très original ?

Armelle Choplin (AC). Ce livre s'inscrit dans la continuité des travaux que nous avions menés avec Olivier Pliez (Directeur de Recherches au CNRS, Montpellier). Depuis une quinzaine d'années, nous nous attachions à suivre des objets de consommation courante globalisés, en particulier des jeans pour Olivier Pliez, et pour moi, des produits de seconde main, comme des réfrigérateurs ou des voitures. En suivant ces objets depuis les lieux de consommation et en remontant la filière jusqu'au lieux de production, nous essayions de rendre compte de la dimension matérielle et de l'insertion de populations pauvres dans une économie globalisée. C'est cette démarche qui a constitué le cœur du livre La Mondialisation des pauvres, que nous avons co-écrit et publié en 2018 au Seuil. Mes terrains d'alors étaient au Sahel (Mauritanie, Mali, Sénégal) et en Afrique du Nord et de l'Est (Maroc, Algérie, Soudan). En arrivant au Bénin en juillet 2016, grâce à une délégation à l'Institut de Recherche pour le Développement, je découvrais Cotonou mais surtout une autre Afrique, celle du Golfe de Guinée, très dense et très urbanisée par rapport au Sahel. J'ai immédiatement eu le désir de comprendre comment l'urbanisation se matérialisait dans cette zone. Et pas seulement au Bénin. J'ai été saisie par cette masse urbaine, la 
plus grande concentration urbaine d'Afrique qui prend forme d'Abidjan à Lagos, ce qu'on appelle le corridor urbain ouest-africain ${ }^{1}$. Pour avoir parcouru intensément ce corridor entre Accra, Lomé, Cotonou, Poro-Novo et Lagos, j'ai été surprise par la couleur gris-ciment qui donne la continuité à cette coulée urbaine qui s'étire sur près de $1000 \mathrm{~km}$. Le ciment est omniprésent dans le paysage et dans les esprits. Il s'est imposé à moi. J'ai décidé de le suivre et de raconter sa vie.

En parallèle, j'avais assisté à une conférence de David Harvey à la conférence RC21 des sociologues urbains en août 2015. Il avait insisté sur la consommation du ciment et du béton en Chine et dans le monde (Harvey, 2016). Ses mots résonnaient dans ma tête lorsque j'arpentais le corridor urbain ouest-africain. Il m'est apparu comme une évidence d'étudier le béton et le ciment dans la fabrique urbaine. Le sac de ciment est emblématique des transformations urbaines, politiques, sociales et économiques des pays du Sud.

Illustration 1 - École Primaire, Cotonou, Bénin

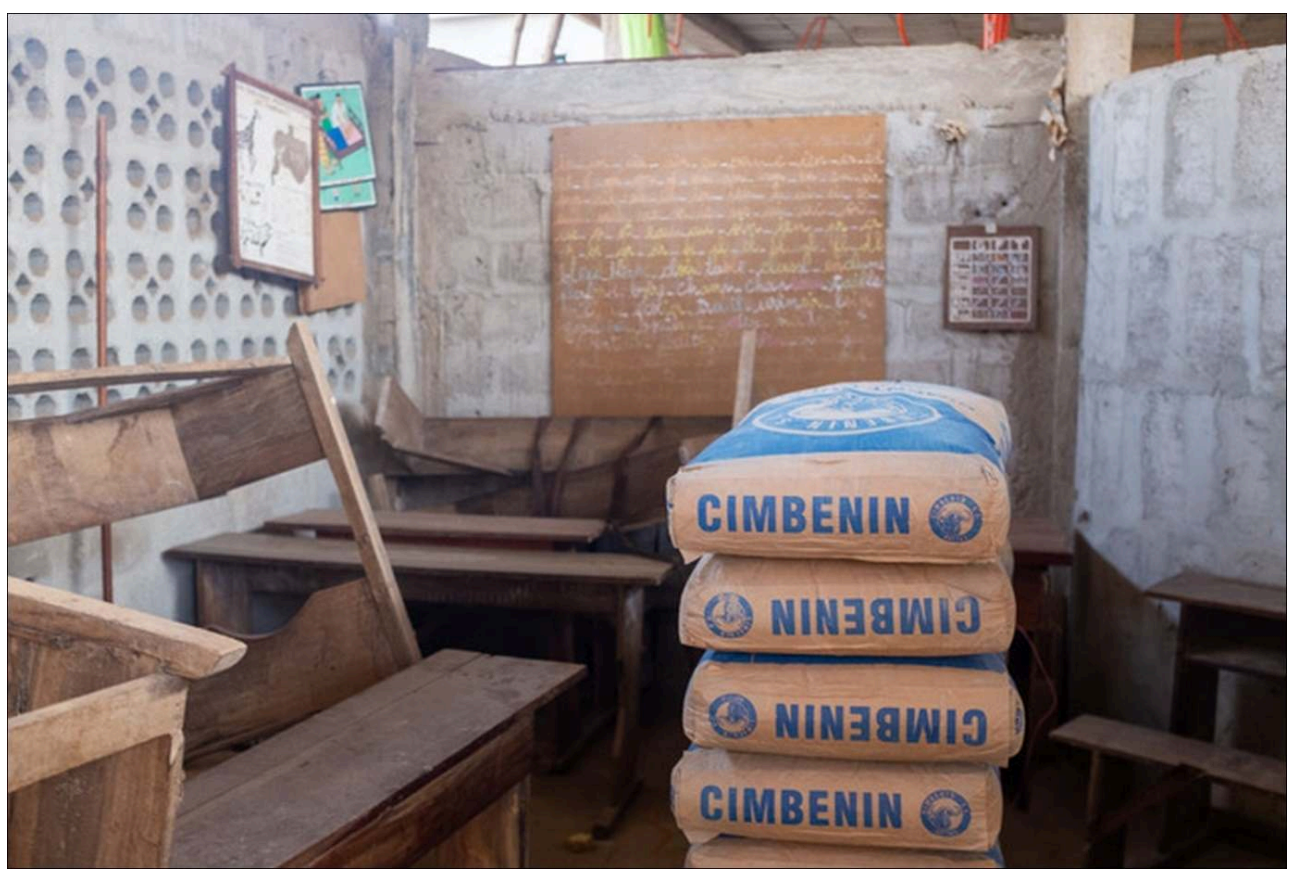

Auteur : A. Choplin, 2018.

\section{SW. Pourquoi plus au Sud qu'au Nord?}

AC. Car les villes des pays du Nord sont déjà saturées en béton : on détruit, on rénove, on reconstruit mais c'est peu. La consommation est déjà à son maximum : elle tourne en moyenne à $500 \mathrm{~kg}$ par habitant et par an. En Afrique, on est à $100 \mathrm{~kg}$ par habitant et par an : il y a une marge de progression énorme. Tout semble encore à construire : logements, routes, barrages, ponts... Et surtout, la demande sociale en béton est très forte. Les habitants ne pensent qu'à construire, à mettre leur famille à l'abri entre quatre murs de béton, à agrandir pour avoir des locataires et donc à acheter des sacs de ciment. On peut d'ailleurs acheter des sacs à n'importe quelle heure du jour et de la nuit. Il y en a à vendre à chaque coin de rue. Le sac de ciment est devenu une source principale de dépense et un indicateur économique du coût de la vie, comme le prix du pain ou d'un paquet de cigarettes. Hommes et femmes, riches et pauvres... tout le monde connait le prix d'un sac de ciment car tout le monde à affaire avec lui. 
Le ciment est chargé de symbole: il est synonyme de modernité mais aussi de réussite. La place d'un individu dans la société se mesure au nombre de mètres cubes qu'il/elle a coulé. Les hommes politiques se font réélire sur des constructions de tours et de grands projets, le béton est au cœur des enjeux politiques. Et parallèlement, les bailleurs de fonds appuient ce secteur comme outil de développement et levier de lutte contre la pauvreté.

SW. C'est toute la filière qui est présente dans l'ouvrage...

AC. J'ai essayé de suivre les sacs de ciment sur les routes, depuis les carrières et usines jusqu'aux chantiers et remonter toute la chaîne de production du béton. Jusqu'aux années 1980-2000, le ciment était un matériau qui renvoyait à la colonisation, importé par les colons. Mais depuis une dizaine d'années, le changement est de taille : le ciment est produit à partir de carrières de calcaires locales. Il faut rappeler le rôle primordial d'un homme en particulier, Aliko Dangote, l'homme le plus riche d'Afrique et $25^{\text {ème }}$ fortune mondiale, qui a ouvert plus d'une dizaine de cimenteries en l'espace de 10 ans dans l'ouest de l'Afrique. Il incarne aujourd'hui la figure de la réussite en Afrique, celui qui a transformé ce produit en un produit $100 \%$ africain. Il a "africanisé » ce matériau colonial. Il concurrence désormais les grandes entreprises européennes, comme le Franco-Suisse LafargeHolcim ou l'Allemand Heidelberg qui avaient un quasi monopole. Le ciment donne lieu à d'intenses circulations: il peut être produit au Nigeria avant d'être revendu au Ghana, parcourant près de $500 \mathrm{~km}$ sur des camions qui voyagent par centaines en convoi. Ces circulations sont le signe d'une géopolitique régionale nouvelle qui laisse entrevoir tout une chaine de production et logistique.

\section{SW. Observe-t-on d'autres transformations économiques?}

AC. Oui. Dangote va créer la première raffinerie de pétrole et ses investissements concernent aussi l'agroalimentaire (sucre, pâtes alimentaires). Ce personnage est symptomatique de la transformation des économies africaines. Avec Dangote et le béton, une nouvelle ère s'ouvre, celle de "l'Africapitalisme». Ce terme créé par le riche homme d'affaire nigérian Tony Elemulu annonce un nouveau stade du capitalisme africain, avec des entreprises et industries africaines qui concurrencent les grands groupes internationaux. Ces hommes - et des femmes - d'affaires se disent philanthropes et souhaitent investir les bénéfices en Afrique, dans leur propres pays. Ils sont à l'image de cette Africa Rising, cette Afrique qui émerge et qui gagne.

SW. Comment s'est déployée votre enquête de terrain?

AC. J'ai repris la méthode dite du Follow the Thing, familière du géographe Ian Cook (2004), pour comprendre la vie sociale du ciment, pour reprendre l'expression de Arjun Appadurai (1986) the Social Life of things. J'ai donc suivi le ciment le long du corridor, selon une approche horizontale. Puis j'ai adoptée une approche verticale, multi-scalaire en rencontrant tous les acteurs de la filière béton: des directeurs de cimenterie jusqu'aux maçons en passant par les promoteurs immobiliers, les entreprises de BTP, les détaillants, à tous les étages et à toutes les échelles. Sur le terrain, je me suis beaucoup rendue au Ghana, au Togo, au Nigeria, tout en vivant au Bénin. Dans les grandes villes, j'ai essaayé d'établir les mêmes grilles d'enquêtes et, à Cotonou, j'ai suivi plusieurs chantiers de construction pendant trois ans. Et j'ai également essayé de cartographier la distribution spatiale de tous les revendeurs de ciment, avec Open Street Map et Kobo Collect, une base de données d'enquêtes en ligne connectée à Open Street Map qui permet de créer des cartes à partir de ses 
propres collectes de données.

Ce livre tente donc de restituer une géographie en mouvement tout en offrant un panorama de l'économie du béton dans des villes ouest-africaines.

Illustration 2 -Carrefour Béton Armé, Calavi, Bénin

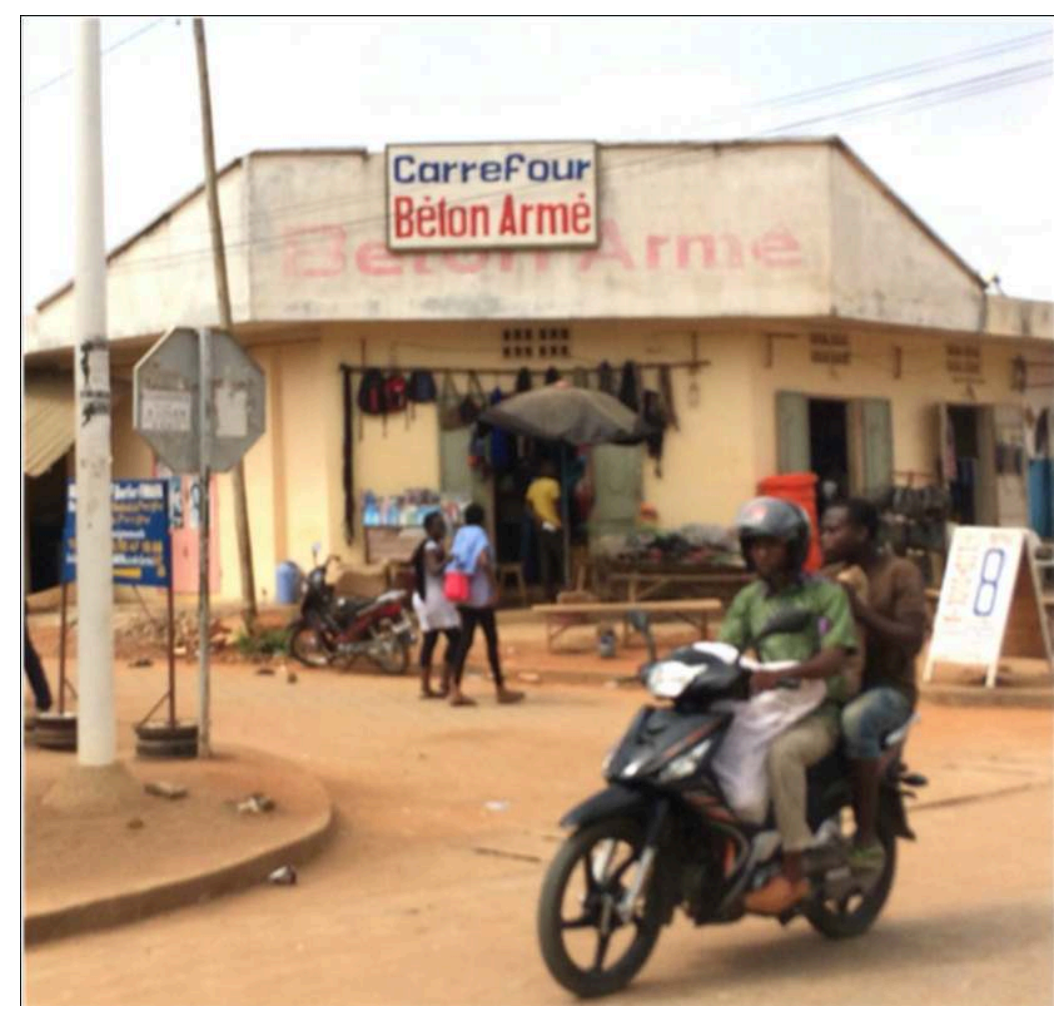

Auteur : A. Choplin, 2018.

SW. Vous consacrez tout un chapitre aux alternatives au béton. Qu'apportent-elles au débat sur la ville durable?

AC. Il faut rappeler qu'il y a un consensus en Afrique sur le ciment. Les bailleurs de fonds appuient la filière béton, les habitants réclament du béton, et les besoins sont énormes, du fait de la croissance démographique. Mais, en parallèle, il commence à y avoir une critique de ce matériau, car il n'est pas adapté au climat: il génère une chaleur insoutenable dans les maisons. Par ailleurs, le béton n'est pas durable, contrairement à ce qu'on croit, il a une durée de vie de 50 à 70 ans. En Europe, les tours et les ponts commencent à s'effondrer, faute d'entretien. Qui va entretenir les équipements en Afrique? Par conséquent, des initiatives émergent et commencent à critiquer le tout béton ${ }^{2}$, résultat de choix politiques et culturels. Or, cela ne peut être la solution, ni en Europe ni en Afrique. Des alternatives pour construire autrement invitent à redécouvrir l'architecture africaine, dont on a longtemps dit, à tort, qu'elle n'existait pas réellement, à repenser la ventilation dans les maisons, à revenir à des matériaux plus durables, comme la terre ou bois là où c'est possible. Ces initiatives montrent que des alternatives sont possibles, y compris en Afrique, et qu'on pourrait s'en inspirer. 
SW. Ce qui vient étayer certaines de vos propositions antérieures qui invitaient à désoccidentaliser la pensée urbaine...

AC. C'est vrai, je continue de croire qu'il faut déplacer le regard, aller voir plus au Sud ce qui se passe car c'est là que s'inventent nos futurs urbains. J'essaie de faire le lien entre des initiatives en Europe et en Afrique. En Suisse, patrie de Le Corbusier et du bunker, il n'est pas aisé de prendre ses distances avec le béton, qui fait partie de la culture locale. Mais des individus, des collectifs essaient de construire en pierre, en bois, et essaient de connecter les réseaux d'un continent à l'autre. Il y a de l'espoir !

SW. Vous évoquez aussi les gravats et tout l'aval de cette production urbaine...

AC. C'est une question qui est de plus en plus importante en Europe, autour du métabolisme urbain, du cycle de vie des matériaux, du recyclage, de la démolition et des gravats (Mongeard, 2017 ; Barles, 2018...). La question se pose avec acuité en Afrique, de quoi mener des études passionnantes sur ces sujets. De même que sur la digitalisation de cette économie, sur le fait qu'on peut acheter très facilement ces matériaux en ligne, avec un simple smartphone, ce qui change les manières de construire. Je souligne d'ailleurs dans le livre le rôle important de la diaspora dans ces relations. Les élites sont à la fois celles qui construisent le plus en béton, des villas imposantes comme celles que l'on peut voir dans les films nigérians de Nollywood, mais aussi celles par qui le changement peut venir, faisant circuler les idées entre Europe, Amérique et Afrique. Aujourd'hui, certaines personnes commencent à souhaiter des maisons en bambou, mieux ventilées, c'est par elles que peut venir le changement, mais aussi par les grands groupes qui sont dejà à même de produire des ciments plus « verts».

\section{SW. Quels sont les effets de ce boom du ciment?}

AC. C'est une des industries les plus polluantes au monde : la fabrication de ciment représente $7 \%$ des émissions de gaz à effet de serre ! Il s'agit d'une industrie très énergivore puisque produire du ciment nécessite de porter le calclaire et l'argile à $1450^{\circ}$. Les cimenteries dotées de leur propre centrale d'énergie, qui fonctionnent au pétrole (nigerian souvent) se multiplient. C'est donc un sujet écologique très sensible, au cœur des enjeux de demain. Au même titre qu'on a eu par le passé la prise de conscience sur les plastiques et l'avion, il y a une prise de conscience récente sur le rôle du BTP pour penser la durabilité.

SW. L'ouvrage montre que cela touche aussi à l'économie du sable.

AC. Avant on prélevait du sable sur le littoral avec les conséquences dramatiques que l'on sait sur l'érosion côtière. Maintenant, cette pratique est interdite, donc on utilise du sable de lagune, cela a des effets profonds sur l'environnement. Il est important de penser à d'autres manières de construire car on sait que les ressources en sable sont menacées, comme le dit très clairement un rapport des Nations Unies pour l'Environnement (UNEP 2019).

SW. J'ai beaucoup apprécié votre clin d'œil au conte des trois petits cochons et du lien entre ciment et promotion sociale.

AC. La "vie sociale du ciment " fait l'objet d'un chapitre central dans le livre. Le béton est au coeur des relations sociales. On offre du ciment en cadeau, on reçoit des sacs de ciment quand on part à la retraite. Il renvoie aussi aux rapports de genre. Rappelons que le BTP est un milieu très masculin et le béton associé à la virilité : " si je construis en dur, je suis un homme fort ». Mais les femmes ne sont pas absentes de 
ce secteur, loin s'en faut : certaines achètent le ciment, les matériaux de construction, connaissent les dosages qui sont indiqués en pictogrammes sur les sacs qui permettent cette diffusion. Certaines femmes construisent par elle-même et supervisent les chantiers, affirmant par là une manière de devenir plus respectées et respectables. Certaines sont à la tête d'empires immobiliers, comme la femme d'affaires Burkinabé Sanata Yaméogo. En Europe, très peu de gens savent couler du béton, en Afrique tout le monde le sait.

Illustration 3 - Chantier, Ladji, Cotonou, Bénin

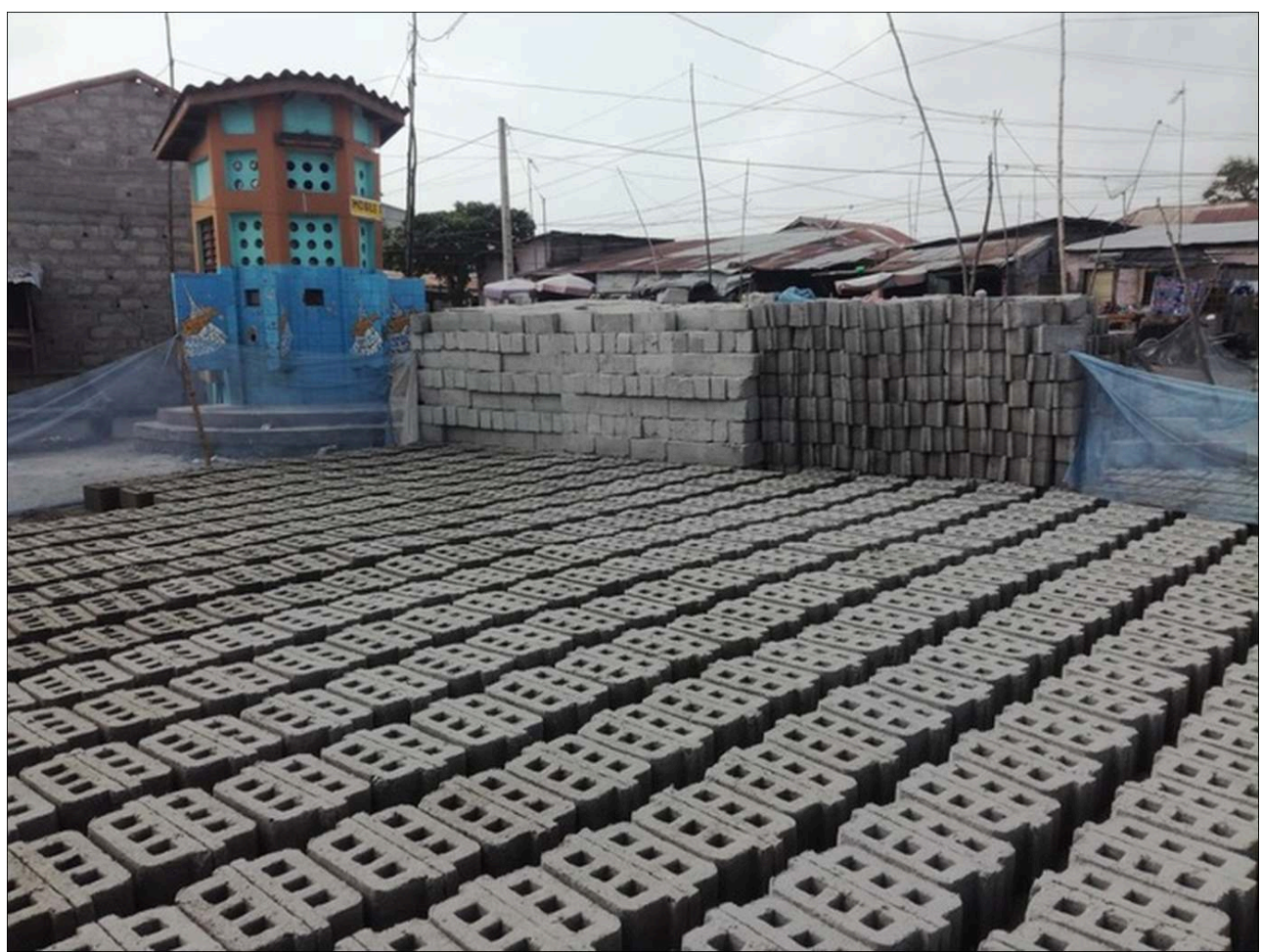

Auteur : A. Choplin, 2018.

SW. Comment se fait lien entre ciment, promotion sociale et formalisation des quartiers autoconstruits?

AC. Il y a plusieurs "villes de béton»: celle des programmes étatiques dits de logements sociaux, même s'ils sont la plupart du temps réservés aux classes moyennes et supérieures, celle des investisseurs étrangers et des grands projets, et celle qui résulte de l'autoconstruction, révélant une urbanisation incrémentale. Le béton participe à la généralisation de l'habitat en dur, même dans les bidonvilles. Le ciment est pour les habitants de ces quartiers une manière de revendiquer leur place dans la ville, c'est un premier pas vers une revendication d'un droit là la ville: j'investis, je construis en béton, j'ai donc droit à faire partie de la ville, à être reconnu comme citadin. Cela prend une dimension réellement politique dans les revendications sociales. Le livre n'est pas un manifeste contre le ciment, mais son but est de comprendre comment le ciment et le béton font partie de l'environnement quotidien des villes africaines contemporaines, qu'on a longtemps vues comme des amas de bidonvilles en tôle et en bois. 
SW. J'ai été saisi par l'intrication du secteur de la construction avec une internationalisation inhabituelle, indienne, libanaise, chinoise notamment...

AC. Tout à fait, et j'espère que cela pourra faire l'objet de prochains terrains. Les matériaux de construction connectent ces villes ouest-africaines avec des espaces inattendus, au Liban, en Inde, en Chine. Cela est lié à des réseaux diasporiques relativement méconnus. C'est notamment très parlant avec l'acier qui, une fois récupéré, est réexpédié vers l'Inde ou la Chine. Il y a sur place des grossistes indiens et chinois qui ont des usines locales de production de fers à béton et d'autres matériaux. D'autres pistes se profilent : les carrelages, qui relient l'Italie, l'Espagne et ces pays, avec l'émergence des classes moyennes et supérieures qui veulent les meilleurs matériaux de décoration. Les marques italiennes ouvrent des showrooms dans les villes africaines. De nouveaux réseaux se sont mis en place assez récemment suite à l'ouverture des marchés ouest-africains. On pense souvent à la Chine mais moins aux Libanais, Indiens et à ces industries européennes. C'est plus nuancé qu'un tout made in China en Afrique.

\section{SW. Pourriez-vous revenir sur le rôle des différents investisseurs?}

AC. Il est intéressant de constater que le même ciment est utilisé pour construire des tours futuristes dans les grands projets (comme, au Nigeria, Eko Atlantic City), avec des investissements privés dont on ne connaît pas bien les circulations - il est compliqué d'investiguer là-dessus -, pour construire les programmes de « logements sociaux » ou pour construire une maison individuelle. C'est le même sac de ciment mais pas le même argent. De nouveaux acteurs souhaitent construire leurs propres villes et créer des espaces privés. Je pense par exemple à Akon, un rappeur sénégalais américain qui a décidé de créer sa ville au sud de Dakar en négociant plusieurs hectares. Cela mériterait des enquêtes approfondies. C'est le début de la financiarisation des villes africaines. Dans ce processus, le béton est justement ce qui permet l'ancrage matériel de ces flux immatériels.

SW. Comment se pose alors la question du raccordement aux réseaux fonctionnels et à la gouvernance urbaine de façon plus générale?

AC. Il n'y a pas toujours les services et équipements, notamment quand les constructions se font dans les périphéries lointaines. J'insiste dans le livre sur le fait qu'on a de l'urbain, mais on n'a pas de la ville. On a des formes urbaines, mais pas d'urbanité et pas de services urbains. Tout est privé : l'électricité, les dispensaires, les écoles. Dans ces périphéries, l'habitat en dur se multiplie, mais rien de ce qui fait la ville. Les cimentiers quant à eux devancent la demande : ils déposent des containers de ciment dans ces franges urbaines. Ils devancent la ville et amènent l'urbain. Par exemple, sur la centaine de kilomètres qui séparent Lomé et Cotonou, tout est quasiment bétonné. On se demande : qui gère ces espaces, alors que ce n'est plus du rural mais pas encore de la ville? De nombreuses communes ont un statut rural. Elles ont peu de revenus. Par conséquent, pour faire rentrer de l'argent, elles proposent de diviser les terrains, de lotir, permettant à des gens de construisent. Ces communes deviennent totalement urbanisées, alors qu'elles n'ont a pas les outils financiers ni administratifs pour gérer ce développement. 


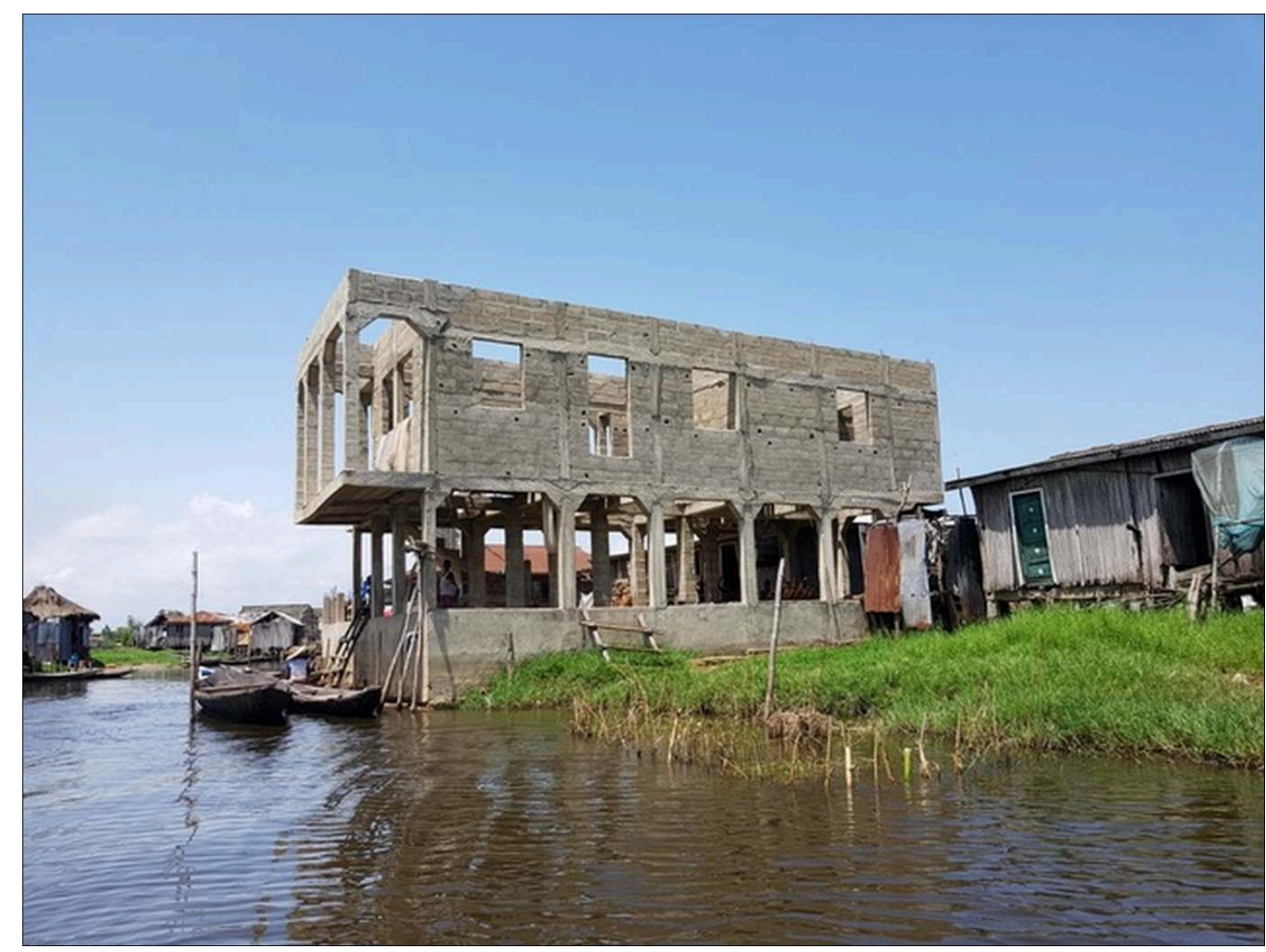

Auteur : A. Choplin, 2018.

\section{SW. Donc cela a des effets politiques ?}

AC. Oui. Cotonou (1,5 millions d'habitants) n'est situé qu'à $30 \mathrm{~km}$ de Porto Novo (600 000 habitants), ce qui occasionne des rivalités : les mairies n'arrivent pas à penser le territoire comme faisant un, elles s'opposent sur des limites administratives. L'intercommunalité n'existe pas comme chez nous, ce qui pose des problèmes importants de gestion. Il faut inventer les outils de régulation, entre villes et même entre pays. Il faut imaginer que Cotonou n'est qu'à $125 \mathrm{~km}$ du centre ville de Lagos, une agglomération de près de 25 millions d'habitants, autant dire que Cotonou fait partie de la banlieue de Lagos. Les bouchons de Lagos commencent à la périphérie de Cotonou!

SW. Les choix d'écriture sont tout à fait originaux et particulièrement agréables à la lecture, c'est un livre très facile à manipuler, et merveilleusement illustré. Malgré son format presque de poche, c'est ce qu'on peut appeler un beau livre en SHS, à un prix dérisoire vu le nombre d'images : c'est assez incroyable et cela suppose un travail pointu de mise en page.

AC. J'aimerais d'abord souligner que le livre est en accès libre, sur le site de l'éditeur et de mon université. C'est une démarche très importante pour que le public africain francophone, et en particulier les étudiant·es, aient accès facilement à ce travail. D'autant plus qu'il est assorti d'une version augmentée en ligne, avec des ressources nombreuses, des vidéos (films, drones). Ce livre est en vérité un double produit, la version papier et la version augmentée en ligne. Dans le travail iconographique, nous avons souhaité, avec l'éditeur genevois MétisPresses, spécialisé dans le champ urbain et l'architecture, et Martin Lozivit, l'assistant de recherche avec qui j'ai travaillé sur le terrain, donner vie au béton et donner à voir ces villes assez méconnues du grand public et souvent assimilées à de nombreux clichés. Le livre propose près de deux cents photos et cartes qui font partie intégrante du choix d'écriture : bien sûr, il y a 
les références académiques, mais le livre raconte la biographie du ciment, teinté de vignettes, d'histoires de vie, d'anecdotes de terrain. J'ai voulu croiser ce travail académique avec d'autres références empruntées à la littérature et au cinéma. D'autant plus que ces deux domaines artistiques sont très riches en ce moment dans ces villes africaines, où foisonnent des productions d'artistes incroyables, qui pour beaucoup rendent compte des transformations urbaines. C'est donc une écriture entre le livre universitaire classique et l'essai lisible par un grand public. Suite à $L a$ mondialisation des pauvres, qui avait eu un grand succès, j'ai souhaité garder ce style d'écriture et format. Le livre, à ma grande surprise rencontre un certain écho. Il circule par exemple dans le milieu des architectes... La version anglaise est sur le point de sortir, chez Wiley, dans la collection IJURR Studies in Urban Social Change. Le livre est intitulé Concrete city: material flows and urbanisation in West Africa.

SW. II y a de belles incursions vers les utopies et les dystopies urbaines...

AC. Il y a une littérature intéressante qui émerge sur l'afrofuturisme, qui donne à voir un futur qui s'imagine dans des villes africaines. Cette littérature, qui relève de la science fiction, a souvent comme point de départ Lagos. Je pense notamment à Nnedi Okorafor (2013), qui a un succès fou. Elle est la grande autrice de science fiction, connue notamment de la jeunesse avec sa série Akata Witch. De même, le film Black Panther, qui se passe en Afrique, montre que le futur urbain peut s'inventer de ces villes de béton au Sud, qui incarnent le futur pour la grande majorité des gens (Guitard, 2018). Je pense encore aux romans de Chimamanda Ngozi Adichie (2016), qui dans Americanah, décrit les transformations de sa ville, Lagos, la spéculation immobilière, les coupures d'électricité...

SW. On se prend à imaginer, après avoir refermé votre livre, que le corridor urbain ouest africain est la capitale de la modernité du XXIe siècle!

AC. Oui ! J'ai même consacré un chapitre à "Walter Benjamin à Lagos », un clin d'œil pour dire que la capitale de la modernité contemporaine n'est plus Paris comme pour Benjamin au début du XX $\mathrm{XX}^{\mathrm{e}}$ siècle, mais bien Lagos en ce début de $\mathrm{XXI}^{\mathrm{e}}$ siècle. J'espère que les lecteurs et lectrices, en refermant l'ouvrage, se diront que nos futurs urbains s'écrivent aussi et surtout dans ces espaces, pour le pire mais aussi je l'espère pour le meilleur.

\section{BIBLIOGRAPHIE}

Adichie C. N., 2016. Americanah. Editions Gallimard.

Appadurai A. (dir.), 1986. The Social Life of Things: Commodities in Cultural Perspective. Londres, New York, Cambridge University Press.

Barles S., 2018. Métabolisme urbain, transitions socio-écologiques et relations ville-campagne. Pour [En ligne sur Cairn], vol. 236, nº 4, p. 49-54. DOI: https://doi.org/10.3917/pour.236.0049

Choplin A., Pliez, O., 2018. La mondialisation des pauvres : loin de Wall Street et de Davos. Paris, Seuil. 
Cook I. et al., 2004. Follow the Thing: Papaya, Antipode, vol. 36, nº 4, p. 642-664.

Guitard É., 2018. Le Wakanda de "Black Panther" : une Afrique du futur en miniature ? Blog de la revue Terrain [En ligne]. URL: https://blogterrain.hypotheses.org/9982

Harvey D., 2016. Abstract from the Concrete. Londres, Sternberg Press.

Mongeard L., 2017. De la démolition à la production de graves recyclées : analyse des logiques de proximité d'une filière dans l'agglomération lyonnaise. Flux, nº 108, p. 64-79.

Okorafor N., 2013. Qui a peur de la mort ? Paris, Le Livre de Poche, coll. Imaginaire.

UNEP (United Nations Environment Programme), 2019. Sand and sustainability: Finding new solutions for environmental governance of global sand resources [En ligne]. Genève. URL: https:// wedocs.unep.org/20.500.11822/28163

\section{NOTES}

1. Pour visualiser cette immense zone urbaine, voir la base de données Africapolis : https:// africapolis.org/fr

2. Cf. Madiana Hazoumé, En finir avec le tout béton, 2017, https://theconversation.com/en-finiravec-le-tout-beton-en-afrique-72556

INDEX

Thèmes : Sur l'Écrit

\section{AUTEURS}

\section{ARMELLE CHOPLIN}

Armelle Choplin, armelle.choplin@unige.ch, est professeur à l'Université de Genève. De 2016 à 2018, elle a été affectée au Bénin en tant que chercheur à l'Institut de Recherche pour le Développement. Elle a récemment publié :

- Choplin A., 2022. Concrete City, Material Flows and Urbanisation in West Africa. WileyBlackwell, IJURR-SUSC Series Book, à paraître.

- Choplin A., Pliez O., 2018. La mondialisation des pauvres, Loin de Wall Street et de Davos. La

République des Idées, Seuil

- Ciavolella R., Choplin A., 2018. Cotonou(s), Histoire d'une ville sans Histoire, Cahiers de la Fondation Zinsou, IRD. 